\title{
An Image Fusion Approach based on Adaptive Fuzzy Logic Model with Local Level Processing
}

\author{
Yashwant Kurmi \\ Maulana Azad National Institute of Technology \\ (MANIT), Bhopal, India
}

\begin{abstract}
Image fusion has lots of application in real life to furnish a combined form of many oriented objects of different images into single image. Adaptive Fuzzy logic model with local level processing is a controlling tool to model image characteristics accurately and been successfully applied to a large number of image processing applications. In this paper an adaptive fuzzy logic model have been proposed with local level processing for fusion of multi-exposure and multi-sensor images. Experimental results demonstrate the superiority of proposed method; it offers approximately 30\%-35\% improvement in Universal image quality index (UIQI) as compared to Marcov Random Field (MRF) fusion method.
\end{abstract}

\section{Keywords}

Adaptive Fuzzy logic, local level processing, Multiresolution decomposition, Multispectral image fusion

\section{INTRODUCTION}

Image fusion is important in many image analysis mechanism in which image data is obtained from multiple sources e.g. multi focus images, multi illumination images. In medical field different types of images having very less part of important information can be fused with different category images to get more information from single fused image. The general purpose of image fusion is to combine relevant information from two or more source images into one single image such that the single image contains as much information from all the source images as possible. The source images involved in such applications can be taken at different times and/or using different sensors. On the analysis of result, source images from different sensors show different physical features. Thus, the fused image is to have a more accurate description of the scene and is, therefore, more useful for human vision or machine perception [1]. In remote sensing applications, there have been a few studies on fusing highresolution panchromatic images and low-resolution multispectral images to improve the spatial resolution [2], [3].

In this paper, we focus on the fusion of images having the same resolution, i.e. multispectral image fusion. A multispectral band covers only a narrow spectral range [3], and different bands represent different aspects of the scene. Multispectral image fusion involves the fusion of several bands in order to improve spectral resolution. The existing image fusion approaches can be classified into three categories: pixel-level, feature-level, and decision-level [4]. This paper is focused on the pixel-level fusion approach. Before image fusion, an image registration algorithm usually needs to be applied in order to align the source images [5]. The basic pixel-level fusion rule includes two steps.

\author{
Vijayshri Chaurasia \\ Maulana Azad National Institute of Technology \\ (MANIT), Bhopal, India
}

- Firstly, it is to be investigated whether a source image contributes to the fused image for each pixel.

- Secondly, the intensity of the pixel in the fused image is obtained from all the contributing source images.

Among the pixel-level fusion rules, two traditional algorithms are; to average the pixel intensities from all the source images or take the maximal pixel intensity among all the source images [16]. The averaging approach is effective in removing the Gaussian noise and increases the signal-to-noise ratio (SNR) but makes the image smoother and results in the loss of contrast information. The maximizing approach can produce the fused image at full contrast but is sensitive to sensor noise [6]. To overcome the limitations of the averaging and maximizing approaches, Sharma et al. [6] gave Bayesian image fusion approach and related it to local principal component analysis. In recent years, multiscale decomposition (MD)-based techniques have been successfully applied to image fusion for different applications such as concealed weapon detection [7] and hyperspectral image fusion [9].

The proposed method is a two level process; firstly the redundant information is minimized from the image by applying the Adaptive fuzzy logic model for global variable processing. Secondly, the Marcov Random Field (MRF) model is used for local level processing of image fusion. The rest of the paper is as follows, section 2 gives the details of proposed method of image fusion. Section 3 covers the result analysis and finally section 4 concludes with some remarks.

\section{PROPOSED ALGORITHMS}

The existing fusion methods are based on the local level processing. In proposed method, Adaptive Fuzzy logic model provides global variable processing to overcome the redundant information field from the source image and provides the space for more informative field to spread over the entire range. In this way Adaptive Fuzzy logic model is a useful tool in image processing and after that MRF model for local level processing is applied. This combination provides better result as compared to the MRF based image fusion model. Figure 1 shows the method more clearly. Here first we describe the Adaptive Fuzzy logic model. That is based on empirical relation of the data analysis of image processing to find the more informative image region very clearly. 


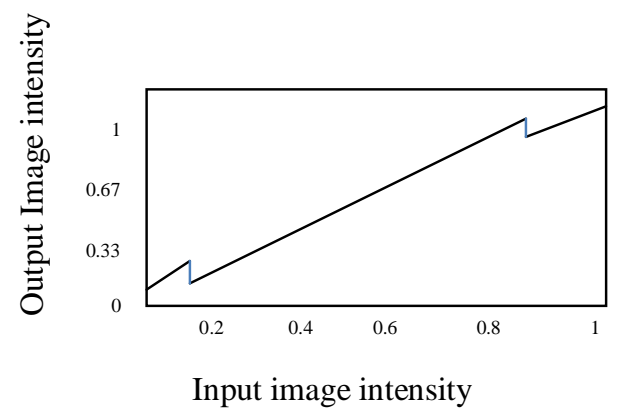

Fig 1: Schematic of the proposed method

The input intensity along horizontal axis higher than $0.95 \mathrm{I}$ is dumped down some lower value and in similar manner the input intensity lower than $0.10 \mathrm{I}$ is pickes up to some higher value by the factor given in equation (1).

$$
\mathrm{G}=\left\{\begin{array}{lr}
0.80 * \mathrm{I}(\mathrm{x}, \mathrm{y}) ; & \text { ifI }(\mathrm{x}, \mathrm{y})>0.95 * \max (\mathrm{I}) \\
\mathrm{I}(\mathrm{x}, \mathrm{y}) ; \quad \text { if } 0.1 * \operatorname{Max}(\mathrm{I})<\mathrm{I}(\mathrm{x}, \mathrm{y})<0.95 * \max (\mathrm{I}) \\
2.5 * \mathrm{I}(\mathrm{x}, \mathrm{y}) ; & \text { ifI }(\mathrm{x} ; \mathrm{y})<0.10 * \max (\mathrm{I})
\end{array}\right.
$$

Where $I(x, y)$ the intensity is image of the concerned image I and $\mathrm{G}$ is the modified image formed by Adaptive Fuzzy logic model. The value of $\mathrm{G}$ is based on the empirical relation with the above coefficients that we find empirically.

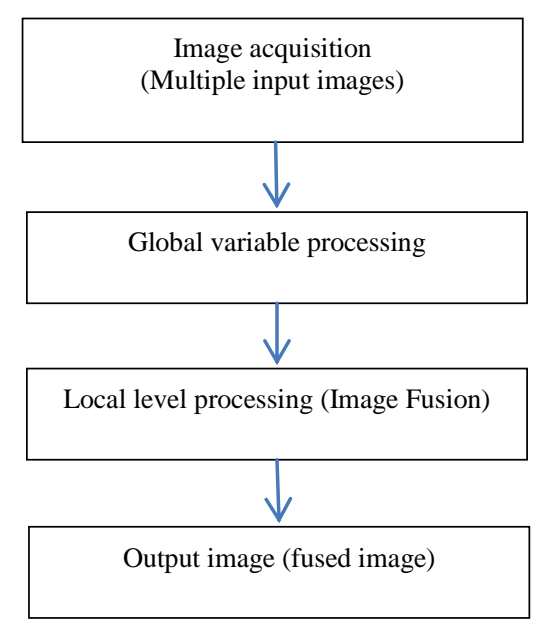

Fig 2: Process flow of the proposed method

\subsection{Image Fusion}

Image fusion is essentially an estimation problem. The objective is to estimate the underlying scene, assuming that each source image contains a good view of only part of the scene [1]. Considering that there are $\mathrm{N}$ source images to fuse. Each source image can be modelled as:

$$
y_{i}(r)=H_{i}(r) x(r)+w_{i}(r), i=1, \ldots, N
$$

where $r$ indicates the spatial coordinates of a pixel, $y_{i}(r)$ is the intensity of the $\mathrm{i}^{\text {th }}$ source image at $\mathrm{r}, \mathrm{x}(\mathrm{r})$ is the intensity of the true scene at $r$ to be estimated, $w_{i}(r)$ is the noise, and $\mathrm{H}_{\mathrm{i}}(\mathrm{r})$ is the sensor selectivity coefficient, taking on values from $\Theta=\{q 1, q 2, \ldots\}$ representing the percentage of the true scene contributing to the $\mathrm{i}^{\text {th }}$ source image [6]. In our work, we use $\Theta=\{0,1\}$, which determines if the true scene contributes to the $\mathrm{i}^{\text {th }}$ source image or not [1]. In the following, for simplicity of notation, "(r)" is omitted. The equation (2) represents the relationship between the source images and the true scene. According to this model, if the true scene contributes to the source image, the source image is modeled as a true scene plus a Gaussian noise. If the true scene does not contribute to the source image, the source image is modeled as Gaussian noise. In practice, particularly in multiple sensor applications and multifocus applications, this model has some limitations. The source images obtained from different sensors sense different aspects of the true scene, and this model may be a coarse approximation in this case. The image fusion problem essentially involves the estimation of $\mathrm{H}_{\mathrm{i}}$ and $\mathrm{x}$. The two traditional algorithms, namely, the averaging and maximizing algorithms, can also be expressed using this model. For the averaging algorithm, $\mathrm{H}_{\mathrm{i}}=1$ for all $\mathrm{i}$. For the maximizing algorithm, $\mathrm{H}_{\mathrm{i}}=1 ; \quad \mathrm{i}=\max \mathrm{i}\{\mathrm{yi}\} ; \mathrm{H}_{\mathrm{i}}=0$, otherwise.

When $\mathrm{H}_{\mathrm{i}}$ is given, the pixel intensity of the fused image can be easily calculated by a Least Squares (LS) technique as [10]

$$
\hat{\mathrm{x}}=(\mathrm{HTH})^{-1} \mathrm{HTY}
$$

Where $\mathrm{H}$ denotes the vector $\left[\mathrm{H}_{1}, \mathrm{H}_{2} \ldots \mathrm{H}_{\mathrm{N}}\right]^{\mathrm{T}}$ and $\mathrm{Y}$ denotes the vector $\left[\mathrm{y}_{1}, \mathrm{y}_{2}, \ldots, \mathrm{y}_{\mathrm{N}}\right]^{\mathrm{T}}$. In practice, we only have the source images available without any prior information and the coefficient $\mathrm{H}$ is usually unknown. According to the LS technique, from the set of all possible values that the coefficient $\mathrm{H}$ can take, the one which produces the highest energy should be selected, i.e.,

$$
\begin{aligned}
\widehat{H} & =\min _{\mathrm{H}}\left\{\mathrm{Y}^{\mathrm{T}} \mathrm{Y}-\left(\mathrm{Y}^{\mathrm{T}} \mathrm{H}\right)\left(\mathrm{H}^{\mathrm{T}} \mathrm{H}\right)^{-1}\left(\left(\mathrm{H}^{\mathrm{T}} \mathrm{Y}\right)\right\}\right. \\
\text { Or } \quad \widehat{H} & =\max _{\mathrm{H}}\left\{\left(\mathrm{Y}^{\mathrm{T}} \mathrm{H}\right)\left(\mathrm{H}^{\mathrm{T}} \mathrm{H}\right)^{-1}\left(\left(\mathrm{H}^{\mathrm{T}} \mathrm{Y}\right)\right\}\right.
\end{aligned}
$$

Note, since $\mathrm{Hi} \in_{\{0,1\} \mathrm{H} \text { has }} 2^{N}$ possible values. Once $\mathrm{H}$ is available, the intensity of the fused image at pixel $\mathrm{r}$ i.e., is obtained by an LS approach [10], which is

$$
\text { 2.1.1.1 } \hat{x}=(H T H)^{-1} H T Y
$$

In the aforementioned model, both the coefficient $\mathrm{H}$ and the intensity of the fused image $\mathrm{x}$ at each pixel are estimated pixel by pixel, and therefore, it is very sensitive to sensor noise. The estimation of the coefficient $\mathrm{H}$ plays an important role in the fusion process. The estimation accuracy of the coefficients directly influences the estimation of the fused image. Since the coefficient $\mathrm{H}$ of a pixel is likely to be similar to the coefficients corresponding to other pixels in its neighborhood due to spatial correlation hence it is estimated by utilizing spatial correlation. A straightforward and simple approach is to assume that the coefficients of pixels within a small window are constant and then select the coefficient which produces the highest energy of pixels within a small window. This strategy has been used in [9]. However, the goal of the LS approach is to minimize the data error $\|y-\hat{y}\|^{2}$, which does not necessarily lead to a small estimation error for either $\mathrm{H}$ or $\mathrm{x}$. A popular strategy for improving the estimation error of LS is to incorporate prior information on $\mathrm{H}$ or $\mathrm{x}$ [11]. Motivated by this fact, the MRF model for local level processing after the adaptive fuzzy logic processing is currently the most effective way to describe the global and local behaviour of both the intensity field and the discontinuity field [12], we propose to employ an FGRF model to estimate the coefficients at Global level and then MRF model for local level processing. It is expected to improve the estimation accuracy of the coefficients $\mathrm{H}$, thereby leading to improved fusion results. 


\section{RESULTS ANALYSIS}

The proposed method is a wide-ranging approach to image fusion and can be applied to different fusion tasks. The fusion algorithm are found to perform well (based on visual assessment) for a variety of tasks without requiring adjustment of the algorithm. Here, the performance of the proposed algorithm is evaluated based on different performance parameters such as variance, Signal-to-Noise Ratio (SNR) and Universal image quality index (UIQI). These parameters help to understand the comparison of proposed method with the MRF fusion method and are associated with the potential functions $V_{c}$ [13]. The model parameters must be estimated for each data set as part of the image processing algorithm. In our algorithm,

(1) The variance $\sigma^{2}$ is assumed as the important parameter in the fusion model. It is estimated the variance by the Maximum Likelihood (ML) standard. It is given by

$$
\text { 3.1.1.1 } \sigma^{2}=\frac{1}{M N} \sum_{i}\left(Y_{i-} H_{i} X\right)^{T}\left(Y_{i-} H_{i} X\right)
$$

The ML estimate of $\mathrm{a}$ is

$$
\text { 3.1.1.2 } \hat{a}=\arg \min _{a} V_{c}(H, a)-\ln Z_{H}
$$

The potential function $V c\left(H_{s} a\right)$ can be simply computed. However, the normalization term $Z_{\mathrm{H}}$ involves a summation of all possible configurations of $\mathrm{H}$, which is practically impossible due to the large computation time. The MPL estimation method is a suboptimal method, which is given by

$$
\text { 3.1.1.3 } \hat{a}=\arg \min _{a} \sum_{s} V_{c}(H(s), a)-\ln Z_{H(s)} . .
$$

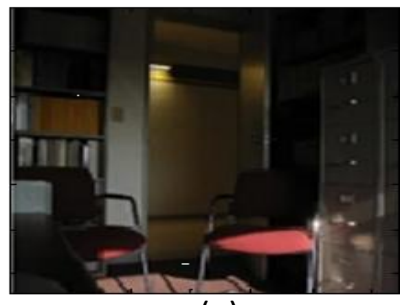

(a)

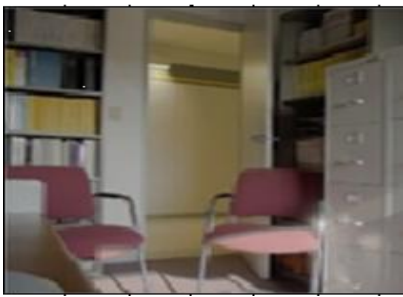

(c)

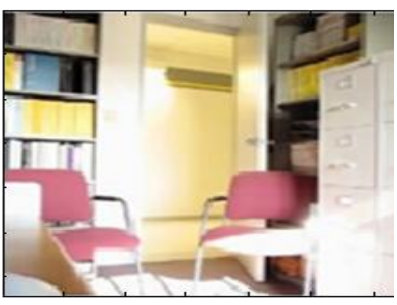

(b)

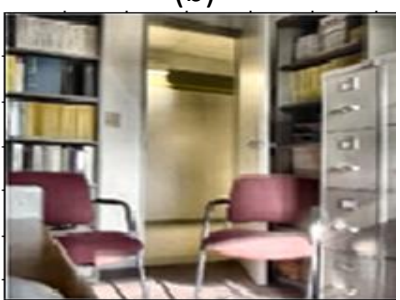

(d)
Fig 3: Results of Multi Exposure Image fusion; (a) Original Image of Library with less exposure (b) Original image of Library with High Exposure (c) Fused Image by MRF method (d) Fused Image by Proposed Method.

(2) SNR: The SNR in decibels, is a direct index to compare the fused image to the reference one [17].

$$
\text { 3.1.1.4 SNR }(Z, \hat{Z})=10 \log _{10} \frac{\sum Z^{2}}{\sum(Z-\hat{Z})^{2}}
$$

(3) Universal image quality index (UIQI): UIQI of two images ( $A$ and $B$ ) is defined as

$$
\text { 3.1.1.5 } Q=\frac{4 \sigma_{A B} \mu_{A} \mu_{B}}{\left(\sigma_{A}^{2}+\sigma_{B}^{2}\right)\left(\mu_{A}^{2}+\mu_{B}^{2}\right)}=\frac{\sigma_{A B}}{\sigma_{A} \sigma_{B}} \cdot \frac{2 \mu_{A} \mu_{B}}{\mu_{A}^{2}+\mu_{B}^{2}} \cdot \frac{2 \sigma_{A} \sigma_{B}}{\sigma_{A}^{2}+\sigma_{B}^{2}}
$$

In figure 3, represents Multi Exposure Image fusion figure 3(a) and 3(b) show the original images of library with less and high exposures respectively, 3(c) shows the MRF fusion method result and 3(d) shows the fusion image of proposed method result. Proposed method gives the better image clarity as we can see, in right part of the image containing handles is very much clearly visible in 3(d) as compared to image 3(c).

TABLE 1. Comparison of parameters for multi illumination Image Fusion

\begin{tabular}{|l|l|l|l|c|}
\hline \multicolumn{1}{|c|}{ Method } & \multicolumn{1}{c|}{ Mean } & \multicolumn{1}{c|}{ Variance } & SNR & UIQI \\
\hline MRF Method & 0.3781 & 0.0251 & 8.2547 & 0.7244 \\
\hline $\begin{array}{l}\text { Proposed } \\
\text { Method }\end{array}$ & 0.5223 & 0.0559 & 7.5762 & 0.9424 \\
\hline
\end{tabular}

Table 1 reperesents the performance comparison of proposed and MRF methods of image fusion. Proposed method improves the UIQI by $30.21 \%$ as that of MRF method due to the global level processing by Adaptive fuzzy logic model.

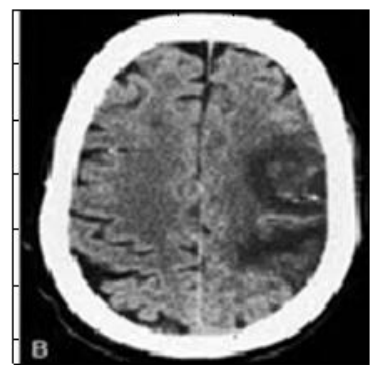

(a)

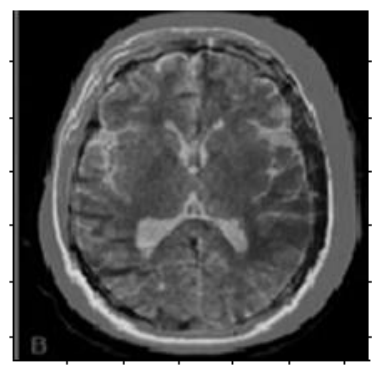

(c)

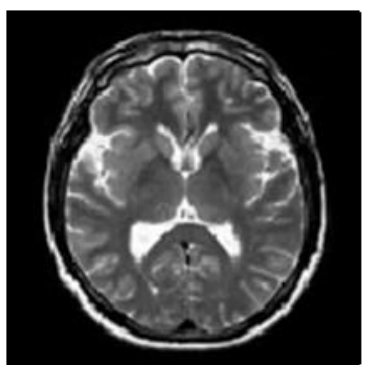

(b)

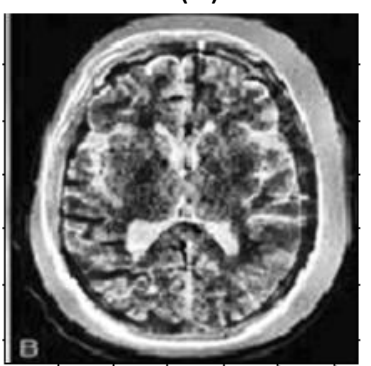

(d)
Fig 4: Results of Multi Sensor (CT scan and MRI of human brain) image fusion; (a) Original CT Scan Image of human Brain top view (b) Original MRI Image of human Brain (same part) top view (c) Fused Image by MRF method (d) Fused Image by Proposed Method.

The Multi-sensor image fusion is shown in figure 4 of two sensors; one is CT scan and other is MRI images of human brain images. Proposed method gives better visible quality of fused image.

TABLE 2. Comparison of parameters for multi-sensor (CT scan and MRI of human brain) Image Fusion

\begin{tabular}{|l|c|l|c|c|}
\hline \multicolumn{1}{|c|}{ Method } & Mean & Variance & SNR & UIQI \\
\hline MRF Method & 0.2713 & 0.0361 & 4.8277 & 8.7546 \\
\hline $\begin{array}{l}\text { Proposed } \\
\text { Method }\end{array}$ & 0.4089 & 0.0901 & 4.6262 & 9.8842 \\
\hline
\end{tabular}

In Table 2, quantitative comparison of proposed method is given with MRF method. As we can see proposed method results $32.97 \%$ higher UIQI than MRF method. 


\section{CONCLUSION}

In this paper, an image fusion technique based on a statistical model is proposed. The approach is based on the fact that the decision making in fusion process has significant correlation within its neighborhood and assumed that it can be modeled as an Adaptive Fuzzy logic model with local level processing. It is evident that the proposed method results better performance as compared to MRF method. The proposed fusion method gives approx. $30 \%$ and $38 \%$ better universal image quality index for multi exposure image of office and for multi sensor (CT scan and MRI of human brain) images respectively.

\section{REFERENCES}

[1] R. S. Blum, 2006 "On multisensor image fusion performance limits from an estimation theory perspective" Inf. Fusion, vol. 7, no. 3, pp. 250-263.

[2] Z. Wang, D. Ziou, C. Armenakis, D. Li, and Q. Li Jun. 2005 "A comparative analysis of image fusion methods" IEEE Trans. Geosci. Remote Sens., vol. 43, no. 6, pp. $1391-1402$.

[3] C. Thomas, T. Ranchin, L. Wald, and J. Chanussot, May 2008 "Synthesis of multispectral images to high spatial resolution: A critical review of fusion methods based on remote sensing physics" IEEE Trans. Geosci. Remote Sens., vol. 46, no. 5, pp. 1301-1312.

[4] C. Pohl and J. van Genderen, 1998 "Multisensor image fusion in remote sensing: Concepts, methods, and applications" Int. J. Remote Sens., vol. 19, no. 5, pp. 823-854.

[5] P. K. Varshney, B. Kumar, M. Xu, A. Drozd, and I. Kasperovich, 2005, "Image registration: A tutorial" In Proc. NATO ASI, Albena, Bulgaria.

[6] R. K. Sharma, T. K. Leen, and M. Pavel, 1999 "Probabilistic image sensor fusion" In Proc. Adv. Neural Inf. Process. Syst. 11, pp. 824-830.

[7] H.-M. Chen, S. Lee, R. Rao, M.-A. Slamani, and P. Varshney, Mar. 2005 "Imaging for concealed weapon detection: A tutorial overview of development in imaging sensors and processing," IEEE Signal Process. Mag., vol. 22, no. 2, pp. 52-61.

[8] Y. Zhang, S. De Backer, and P. Scheunders, Nov. 2009, "Noise-resistant wavelet based Bayesian fusion of multispectral and hyperspectral images," IEEE Trans. Geosci. Remote Sens., vol. 47, no. 11, pp. 3834-3843.

[9] J. Yang and R. Blum, 2002, "A statistical signal processing approach to image fusion for concealed weapon detection" In Proc. IEEE Int. Conf. Image Process., , pp. 513-516.

[10] S. M. Kay, 1993, "Fundamentals of Statistical Signal Processing: Estimation Theory". Upper Saddle River, NJ: Prentice-Hall.

[11] Y. C. Eldar, A. Beck, and M. Teboulle, 2007 "Bounded error estimation: A Chebyshev center approach. In Proc. 2nd IEEE Int. Workshop Comput. Adv. Multi-Sensor Adapt. Process, pp. 205-208,

[12] P. Bremaud, M. Chains, 1999, "Gibbs Fields, Monte Carlo Simulation, and Queues", New York: SpringerVerlag,.

[13] H. Derin and H. Elliott, Jan. 1987 "Modeling and segmentation of noisy and textured images using Fuzzy Gibbs Random Fields". IEEE Trans. Pattern Anal. Mach. Intell., vol. PAMI-9, no. 1, pp. 39-55,.

[14] T. Kasetkasem, Dec., 2002 "Image analysis methods based on Markov random field models" Ph.D. dissertation, Syracuse Univ., Syracuse, NY.

[15] J. Besag, 1986 “ On the statistical analysis of dirty pictures” J. R. Stat. Soc., vol. 48, no. 3, pp. 259-302,.

[16] M. Xu,, H. Chen, and P. K. Varshney, Dec. 2011, “An Image Fusion Approach Based on Markov Random Fields". IEEE Transactions On Geoscience And Remote Sensing, Vol. 49, No. 12.

[17] Y. Zhang, S. De Backer, and P. Scheunders, Nov. 2009, "Noise-Resistant Wavelet-Based Bayesian Fusion of Multispectral and Hyperspectral Images", IEEE Transactions On Geoscience And Remote Sensing, Vol. 47, No. 11. 